\title{
Molecular Cloning, Expression, and Function of Synechocystis PCC6803 Type II Peroxiredoxin (sll1621) Gene in Escherichia coli Cells under Salinity Stress Conditions
}

\author{
Ahmed Gaber ${ }^{1,5 *}(\mathbb{D})$, Mostafa M. Farag ${ }^{2,6}(\mathbb{D})$, Ayman K. Ismail ${ }^{2,7}$, Mohamed H. \\ Hassan $^{1,8}\left(\mathbb{D}\right.$, Saqer Alotaibi ${ }^{3}$ a and Walaa F. Alsanie ${ }^{4}$ (D) \\ ${ }^{1}$ Department of Biology, Faculty of Science, ${ }^{2}$ College of Medicine, ${ }^{3}$ Department of Biotechnology, Faculty of \\ Sciences, ${ }^{4}$ Department of Clinical Laboratories, Faculty of Applied Medical Sciences, Taif University, Taif, Kingdom \\ of Saudi Arabia. ${ }^{5}$ Department of Genetics, Faculty of Agriculture, ${ }^{6}$ Department of Biochemistry, Cairo University, \\ Egypt. ${ }^{7}$ Suez Canal University, Forensic Medicine and toxicology Department, Egypt. ${ }^{8}$ Department of Genetics, \\ Faculty of Agriculture, Menoufiya University, Egypt.
}

\begin{abstract}
Microorganism's cycle exposure to reactive oxidants from internal metabolism and abiotic stress conditions, e.g. oxidative stress, salinity, drought, low temperature, high temperature, and high light. Synechocystis PCC6803 genomes typically encode different types of antioxidant scavenging enzymes including Peroxiredoxins (Prxs). There are five genes similar to Prxs were found inthe Synechocystis PCC 6803 genome. Based on sequence homology analysis of Synechocystis PCC 6803s/l1621 gene, it is categorized into type II Prx (PrxII). The presumed amino acid sequence of Synechocystis PCC6803 PrxII protein exhibited identity about $44 \%-99 \%$ to other Prxll proteins from human, plants, algae, and other different cyanobacterial cells. In the last decade, the genetically controllable model organism $E$. coli has been introduced as a viable biotechnological model for genetically modified microorganisms. The Synechocystis PCC6803 sll1621 gene was overexpressed in PTYB21 expression vector and the resulting was named as $p T Y B 21 / s / l 1621$. The $p T Y B 21 / s / 1621$ was overexpressed in Escherichia coli BL21 (DE3) host cell. The overexpressed protein of Prxll gave the recombinant $E$. coli cells the ability to survive under high concentrations of salinity stress, whereas the viability of wild type cells was completely inhibited at the same high concentrations of salinity stress. In conclusion, the present research documented the expressing of the sll1621 gene into $E$. coli cells. This result approved the absence of species barrier in relations to the function of Synechocystis PCC 6803 Prxll protein in different microorganism.
\end{abstract}

Keywords: Synechocystis PCC6803, sll1621,Prxll, salinity stress, E. coli

*Correspondence: agaber60@yahoo.com; +966530741541

(Received: April 08, 2020; accepted: May 20, 2020)

Citation: Gaber A, Farag MM, Ismail AK, Hassan MH, Alotaibi S, Alsanie WF. Molecular Cloning, Expression, and Function of Synechocystis PCC6803 Type II Peroxiredoxin (s/l1621) Gene in Escherichia coli Cells under Salinity Stress Conditions. J Pure Appl Microbiol. 2020;14(2):1195-1202. doi: 10.22207/JPAM.14.2.14

(C) The Author(s) 2020. Open Access. This article is distributed under the terms of the Creative Commons Attribution 4.0 International License which permits unrestricted use, sharing, distribution, and reproduction in any medium, provided you give appropriate credit to the original author(s) and the source, provide a link to the Creative Commons license, and indicate if changes were made. 


\section{INTRODUCTION}

Peroxiredoxins (Prxs) are a universal group of antioxidant proteins that promote the scavenging of several hydroperoxides ${ }^{1}$. In the past decades, Prxs have gotten extensive consideration as a group of thiol-specific antioxidant proteins. Additionally, they named as thioredoxin peroxidases and/or alkylhydroperoxide reductase proteins ${ }^{2}$. It started back to 1989 when an alkyl hydroperoxide reductase activity was isolated from Salmonellatyphimurium and E. coli. The enzyme was able to decrease cumene hydroperoxide as an electron acceptor with NADH or NADPH as an electron donor ${ }^{3}$. Yet, in a way autonomous of selenium, glutathione and heme can replace NADH and NADPH4. Thus, in such situation, two enzymes named AhpF ( $52 \mathrm{kDa}$ ) and AhpC ( $22 \mathrm{kDa}$ ) correspondingly made the activity ${ }^{4}$. According to their genetic background and catalytic activities, there are four different groups of enzymes that belong to Prxs which are 1-Cys Prx, 2-Cys Prx, Prxll, and $\operatorname{PrxQ}^{5}$.

Synechocystis PCC 6803 contains five Prxs genes ${ }^{6}$. These genes are a part of each reported groups as sll1621 (Prxll), sll0755 (2-Cys Prx), slr1198 (1-Cys Prx), and slr0242, sll0221 (Prx Q) $)^{6}$. Investigations of cyanobacterial Prxs mutant strains propose that the physiological roles of these proteins are focused on the adjustment of the growth of the cyanobacterial cells at high light intensities, however the catalytic activity may not generally include peroxide detoxification ${ }^{1,7}$. Interestingly, Synechocystis PCC 6803 Prxll disrupting mutant strain showed a seriously diminished development and growth rate comparative to the growth rate of wild type cells under even normal light intensities ${ }^{1,7}$. Interestingly, a mutant strain of Anabaena PCC 7120 was failed to express one of its four PrxQ, displayed a slow growth rate at moderate light intensities and was hypersensitive to methyl viologen ${ }^{8}$. Perez-Perez et al. ${ }^{9}$ stated that all five enzymes of Synechocystis PCC 6803 Prxs could utilize thioredoxins as an electron donor. The most noteworthy catalytic ability was acquired for the two enzymes of Prxll and TrxQ with thioredoxin as an electron donor?. Kobayashi et al. ${ }^{7}$ indicated that the expression level of Synechocystis PCC6803 sll1621 gene was significantly induced under oxidative stress through the treatment of Synechocystis PCC6803 wild-type cells to methyl viologen for $15 \mathrm{~min}$ under high light intensities. Additionally, numerous defensive mechanism genes are liable for adaptation under a biotic stress conditions in Synechocystis PCC6803 cells. For example, 1-Cys Prx (s/r1198) and Prxll (sll1621) genes are overexpressed under highlight treatment ${ }^{10}$. Also,another antioxidant enzyme in Synechocystis PCC6803, NADPH-dependent glutathione peroxidase-like protein (s/r1992), was found to be overexpressed and enhance the transgenic Arabidopsis plants under several abiotic stress including salinity, drought, chilling and highlight ${ }^{11,12}$. Additionally, it was reported that the mRNA levels of sll1621 under conditions of paraqout-induced oxidative stress increased seven times more than the untreated cells ${ }^{13}$. All these data clearly show the importance of the s/l1621 gene to scavenge the free radicals of reactive oxygen species (ROS) particularly under different abiotic stress conditions such as the exposure to high light and methyl viologen.

The aim of the present researchis to further analyze the effect of the overexpression of the Synechocystis PCC 6803 Prxll protein on the growth of $E$. coli BL21 cells under salt stress condition that induced oxidative stress. The present data will help to more emphasizing of the physiological role of Synechocystis PCC 6803 Prxll under salt stress and its use for the scavenging of ROS that generated under salt stress.

\section{MATERIALS AND METHODS}

\section{Chemicals and bacterial strains}

Ampicillin, X-gal and IPTG purchased from UFC Biotechnology (USA). The restriction enzyme $E c o R I$ and $E$. coli BL21 strain were acquired from NEB (USA). Other different synthetic compounds were obtained with the highest caliber financially accessible. Cloning vector (pGEM-T easy) was bought from Promega (USA); whereas, the expression vector pTVB21 was gotten from NEB (USA).

\section{Growth conditions}

Synechocystis sp. PCC 6803 cells was grown in Allen's medium 14 under dim light condition with shaking for five to seven days. Cloning $E$. coli host cell $(\mathrm{DH} 5 \alpha)$ and the expression E. coli host cells (BL21 DE3) were cultured in LB broth media at $36^{\circ} \mathrm{C}$. Cells harboring recombinant plasmid was cultured and kept on LB media 
accompanied with ampicillin (100 mg/mL). Gene expression of Synechocystis PCC6803 sll1621 in E. coli

Total DNA of Synechocystis PCC6803 cells was isolated as described previously ${ }^{15}$. The specific sll1621 gene was isolated using PCR with the following primer: 5'-CATATGACCCCCGAACGAGTTCC-3' (forward primer) and 5'-CTCGAGTTAGCCG-ACAAAAGCTTTAACG-3' (reverse primer). Both primers were designed to introduce restriction enzymes sites of $\mathrm{Ndel}$ in forward primer and Xhol in reverse primer. The purified PCR product was successfully cloned into E. coli DH5a host cells (Promega, USA) using pGEM-T easy vector according to the instruction of the supplier. The positive transformed colony was confirmed by PCR and DNA sequencer. Next, the confirmed sequenced gene was over expressed in E. coli BL21 (DE3) (NEB, USA) host cell using $p$ TYB21 vector (NEB, USA). The resulting construct was designated as $p T Y B 21 / s / l 1621$.

\section{Production of the recombinant protein}

The recombinant $p T Y B 21 / s / 1621$ vector was cultured in five $\mathrm{mL} L B$ broth accompanied with ampicillin $(100 \mathrm{mg} / \mathrm{mL})$ at two different temperatures $\left(16^{\circ} \mathrm{C}\right.$ or $\left.36^{\circ} \mathrm{C}\right)$, then left overnight. Next, the overnight cultures were inoculated to a new $100 \mathrm{~mL}$ of LB broth. When the $\mathrm{OD}_{600}=0.4$, IPTG $(400 \mathrm{mmol} / \mathrm{L})$ was added and the culture cells were grown for additional three hours to induce the recombinant protein. In parallel, wild type strain harboring empty $p T Y B 21$ vector was cultured. Both cells were harvested, and SDS-PAGE was achieved in $12 \%(\mathrm{w} / \mathrm{v})$ as descripted before ${ }^{14}$. Evaluate of salt stress tolerance of recombinant E. coli cells

The recombinant ( $p$ TYB21/s/l1621) and wild type ( $p$ TYB21/empty) cells were cultured in 50 $\mathrm{mL} L B$ broth accompanied with ampicillin $(100 \mathrm{mg} /$ $\mathrm{mL}$ ) at $36^{\circ} \mathrm{C}$ with shaking. After the induction of the recombinant protein, both cells were collected by centrifugation at $5000 \mathrm{rpm} / 10 \mathrm{~min}$. Next, the collected cells were re-inoculated in five $\mathrm{ml}$ of $L B$ broth and distributed by equal concentration on LB agar medium including several concentrations of $\mathrm{NaCl}(0,400,800$, and $1000 \mathrm{mM})$ and were grown overnight at $36^{\circ} \mathrm{C}$.

\section{Protein sequence evaluation}

The amino acids sequence of Synechocystis PCC 6803 Prxllwas alignment with other Prxllproteins from different organisms using ClustalW program ${ }^{16}$. The molecular information of Prxll protein (amino acids composition, molecular weight and $\mathrm{pl}$ value)wereestimatedusing the ProtParam resourcesfrom ExPASy website as described previously ${ }^{15}$.

\section{RESULTS AND DISCUSSION Characterization of the sll1621 gene}

To defend the cells and organ against oxidative stress and ROS, aerobic organisms have developed a profoundly classy and complex antioxidant prevention system ${ }^{17}$. It includes a diversity of components, both endogenous and exogenous in source. Both components work collaboratively to remove free radicals ${ }^{17}$. Type II Prxs are one of the most important peroxiredoxins proteins that can catalyze the reduction of various hydroperoxides ${ }^{1,2}$. Investigations of cyanobacterial Prxs mutant cells propose that these proteins work in adjustment to growth and development of cyanobacteria at high-light intensity, however the catalytic activity may not generally contain peroxide detoxification ${ }^{1}$. It was found that the interruption of the sll1621 gene had a reduction consequence on the survival growth rate of Synechocystis PCC 6803 cells under normal or dim light circumstances, suggesting that Prxll enzyme is important for the viability of this cells7. The purpose of the present research is to further explore the effect of the overexpression of Synechocystis PCC6803 sll1621 genein E. coli

Table 1. Identity and similarity percent of type II Peroxiredoxin proteins of Synechocystis PCC 6803 with other type II peroxiredoxin proteins

\begin{tabular}{lcccccc}
\hline \multirow{2}{*}{ Type II } & \multicolumn{6}{c}{ Prx protein from Synechocystis PCC 6803 } \\
\cline { 2 - 7 } & S. PCC 6714 & S. PCC 7003 & C. reinhardtii & Jatropha curcas & Oryza sativa & Homo sapiens \\
\hline Identity & $99 \%$ & $85 \%$ & $48 \%$ & $48 \%$ & $43.4 \%$ & $44.2 \%$ \\
Similarity & $99 \%$ & $91 \%$ & $66 \%$ & $66 \%$ & $60.7 \%$ & $62.8 \%$ \\
\hline Journal of Pure and Applied Microbiology & \multicolumn{2}{c}{1197} & & www.microbiologyjournal.org
\end{tabular}


residue separated from the first one by the same number of amino acid residues (Fig. 1) ${ }^{18}$.

According to the phylogenetic tree analysis of Prxll proteins (Fig. 2), the Synechocystis PCC 6803 Prxll was clustered in same group with the similar Prxll proteins from other prokaryotic algae, including Synechocystis PCC 6714 and Synechococcus PCC 7003. The other Prxll proteins from eukaryotic algae, plants and human were clustered together in second group. Among all of these proteins, Synechocystis PCC 6803 Prxll was most nearly to Prxll from Synechocystis PCC 6714 (Fig. 2). The Prxll proteins of plants, Oryza sativa and Jatropha curcas, have an $\mathrm{N}$-terminal extension about 38 amino acids than Synechocystis PCC 6803 Prxll (Fig. 1). Also, the human Prxll protein have an $\mathrm{N}$-terminal about 25 amino acids than Synechocystis PCC 6803 Prxll. These extensions in plants or human Prxll proteins might be coded for a transit peptide anticipated to address the protein to chloroplast or mitochondria, respectively.

Protein expression of Prxll in E. coli cells

We amplify the sll1621 gene using PCR and the resulted specific product was used for cloning in E. coli DH5 $\alpha$ cells (Fig. 3). Thesequence of the PCR product (570 base pair, Fig. 3A lane 1) was checkedusing an automated DNA sequencer apparatus. The results of DNA alignments of the gene productwith other genes in NCBI website indicated that the gene was $100 \%$ homologous to Synechocystis PCC 6803 sll1621 gene (data not shown). To further ensure about the full sequence of sll1621 gene, we performed a restriction gene map using EcoRI restriction enzyme. As expected, the restriction map of the s/l1621 after cutting with EcoRI was given three fragments with lengths of 259 bp, 229 bp and 123 bp(Fig. 3A, lane 2).

The restriction sites of Ndel and Xhol that involved in the gene product were used to overexpress s/l1621 gene into $p T Y B 21$ vector. The resulting was namedaspTYB21/s/l1621, which was transformed into E. coli BL21 (DE3) cells to obtain there combinant protein. Subsequently, we examined the best conditions for the growth of BL21 (DE3) cells to produce Prxll protein with high expression (Fig. 3B). In this case two types of temperatures were used $\left(15^{\circ} \mathrm{C}\right.$ and $\left.36^{\circ} \mathrm{C}\right)$. The reason for choosing $15^{\circ} \mathrm{C}$, as it is recommended for the overexpression of the Intein protein, of the Saccharomyces cerevisiae, that fused at the $\mathrm{N}$-terminus of $p$ TYB21 vector $(56 \mathrm{kDa})$. The recombinant protein was successfully expressed with a sufficient level in BL21 (DE3) cells at $36^{\circ} \mathrm{C}$ (Fig. 3B). The predicted molecular weight of

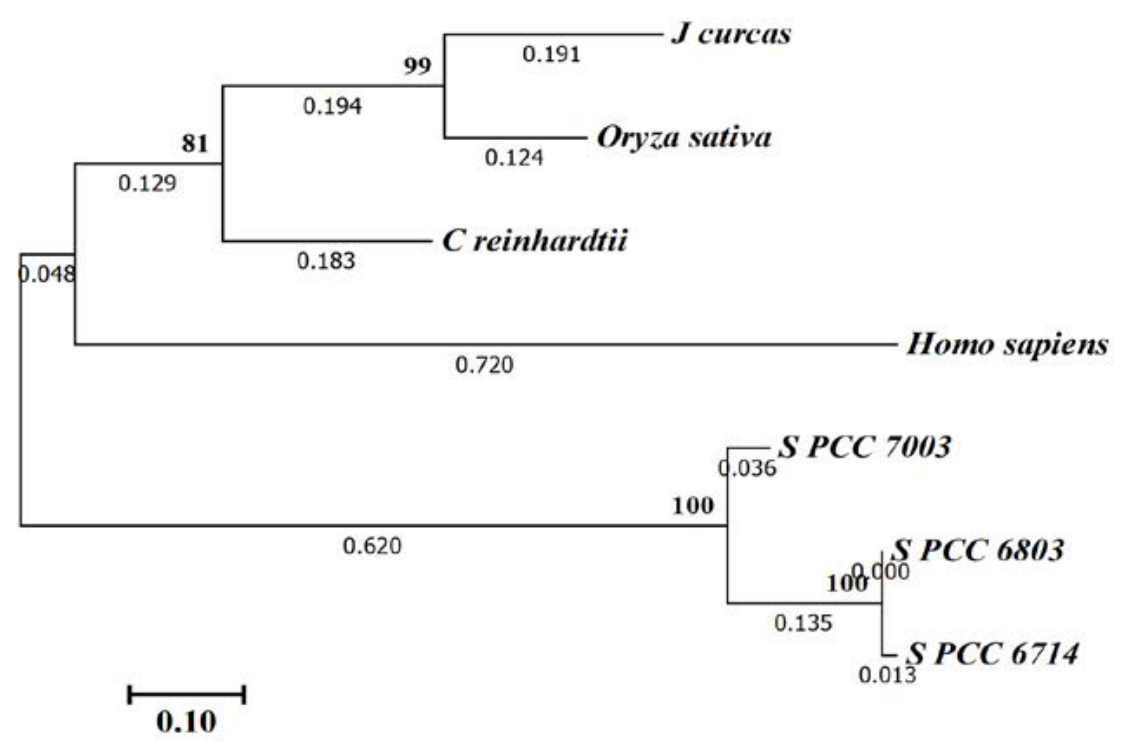

Fig. 2. Molecular phylogenetic tree by Maximum Likelihood method of the Synechocystis PCC 6803 type II Prx protein (S. PCC 6803) with those of the type II Prx proteins from other organisms including Synechocystis PCC 6714 (S. PCC 6714), Synechococcus PCC 7003 (S. PCC 7003), Chlamydomonas reinhardtii, Jatropha curcas, Oryza sativa, and Homo sapiens. The bootstrap consensus tree was from 1000 replicates. Numbers by nodes indicate Maximum Likelihood bootstrap. Evolutionary analyses were conducted in MEGA728. 
the recombinant Prxll protein $(21.167 \mathrm{kDa})$ was detected (Fig. 3B, lane 3). Whereas, we could not detect any protein band related to Prxll molecular weight in E. coli control cells (Fig.3B, lane 4). Interestingly, at $15^{\circ} \mathrm{C}$, we detect a protein with a molecular weight of $77 \mathrm{kDa}$ in recombinant
BL21 (DE3) cells that harboring $p T Y B 21 / s / 1621$ vector (Fig. 3B, lane 2). This molecular weight is considered for the total molecular weight of the Intein protein (56 KDa) that expressed by pTYB21 at $15^{\circ} \mathrm{C}$ plus Prxll protein (21.16 KDa).

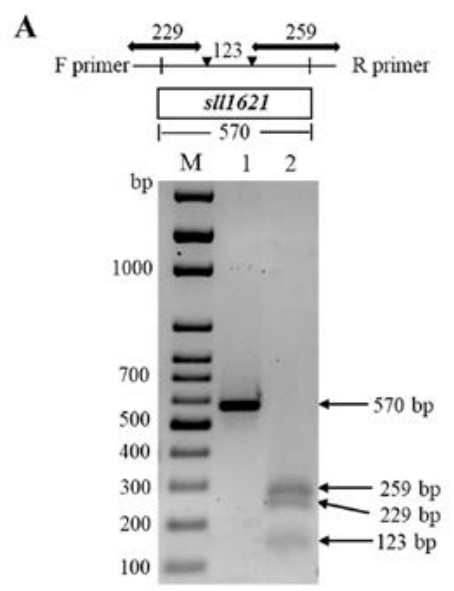

B

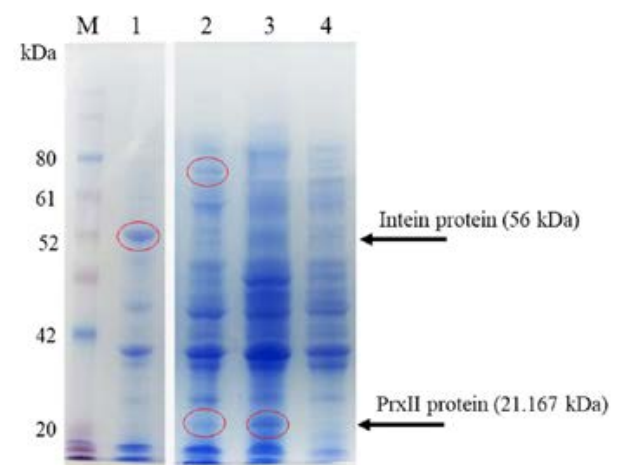

Fig. 3. Isolation and expression of sll1621 gene in E. coli BL21 (DE3) cells. (A) PCR amplification of sll1621 gene that isolated from the genomic Synechocystis PCC 6803 (lane 1), while, lane 2 represented the restriction map of the sll1621 gene using EcoRI. (B) SDS-PAGE analysis of the recombinant Prxll protein after addition of IPTG. Lane 1, E. coli cells transformed with $p T Y B 21 /$ empty vector that cultured at $15^{\circ} \mathrm{C}$ for 16 hours; lane $2, E$. coli cells transformed with pTYB21/sll1621vector that cultured at $15^{\circ} \mathrm{C}$ for 16 hours; lane 3, E. coli cells transformed with $p T Y B 21 / s / l 1621$ vector that cultured at $36^{\circ} \mathrm{C}$ for 3 hours; lane 4, E. coli cells transformed with $p T Y B 21 /$ empty vector that cultured at $36^{\circ} \mathrm{C}$ for 3 hours; lane M, PiNK plus prestained protein ladder (GeneDireX).

\section{Synechocystis PCC 6803 Prxll protein is necessary} for improve growth of $E$. coli under salt stress

Peroxiredoxins are proteins that have molecular weight around 20 to $30 \mathrm{kDa}$ and widely spread in living organisms ${ }^{19}$. Initially, Prxs were identified according to its ability to protect cells especially proteins from abiotic stress particularly oxidative damage that leads to an increase of ROS ${ }^{20}$. Previously, Prxs was termed as the "protector protein" or "thiol-specific antioxidant" before being name again as Prxs ${ }^{21-}$ 24. Five different Prxs genes including s/l1621 were found in the genome of Synechocystis PCC 68036. Also, Kobayashi et al. ${ }^{7}$ stated that the mRNA level of the sll1621 gene is remarkably up-regulated when Synechocystis PCC 6803 are exposed to methylviologen with light conditions. Also, Hosoya-Matsuda et al. ${ }^{1}$ concluded that Prxll is significantly important as scavenging ROS enzyme. They found that the growth of the knockout mutant cells lacking s/l1621 gene was remarkably weak against oxidative stress ${ }^{1}$.
Oxidative injury to microorganism cells frequently happens under different abiotic stress conditions during bioprocess ${ }^{11,15}$. Thus, it has been addressed either the peroxiredoxin activity of Prxll protein assumes a significant useful role in recombinant $E$. coli, and additionally whether such activity might be useful under salinity stress. In the present research, we used the model microorganism, E. coli, to examine the ability of Synechocystis PCC 6803 sll1621 gene to increase the viability of growth of $E$. coli recombinant cells under salt stress. Salt stress was generated in-vitro by culturing the recombinant and wild type $E$. coli cells on LB agar provided with various concentrations of $\mathrm{NaCl}$ (Fig. 4). The $p T Y B 21 /$ empty cells were sensitive to $400 \mathrm{mM} \mathrm{NaCl}$, while, the growth was completely deleted in the presence of $800 \mathrm{mM}$ and $1000 \mathrm{mM}$ of $\mathrm{NaCl}$ (Fig. 4). On the other hand, the recombinant $p T Y B 21 / s / l 1621$ cells increased the growth efficiency under the high concentration of $\mathrm{NaCl}(1000 \mathrm{mM})$, demonstrating that the growth was, for some reason, improved 
compare to those of the $p T Y B 21 /$ emptycells (Fig 4). Accordingly, this survival test under different concentration of $\mathrm{NaCl}$ showed that the s/l1621 gene from Synechocystis PCC 6803 could function as an antioxidant protector enzyme to defend cells from oxidative damage under salt stress (Fig. 4). Similar results documented that the expression of Prxllgene are typically affected by oxidative stress and abiotic stress that caused by hyperoxia, peroxides, UV, and ionizing radiation ${ }^{25-27}$.

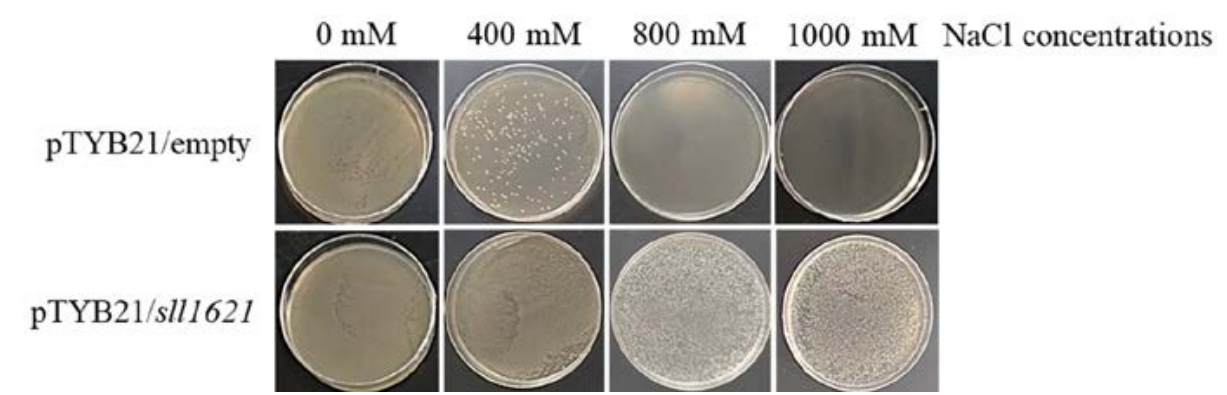

Fig. 4. Impact of various concentrations of $\mathrm{NaCl}$ on the growth of recombinant $p T Y B 21 / s / 1621$ and $p T Y B 21 /$ empty E. coli cells. Full conditions for experiments are depicted in "Materials and Methods section".

\section{CONCLUSIONS}

In the present work, the Synechocystis PCC 6803 sll1621 gene was successfully over expressed in the cytoplasm of E. coli BL21 (DE3) cells. The over expressed protein was verified by SDS-PAGE and observ ableviability test. The present data specify that Synechocystis PCC 6803 sll1621 gene confers tolerance of recombinant $E$. coli cells to high concentration of salt stress. Consequently, these results are strong evidence for the success of gene expression and the absence of species barrier among different microorganisms.

\section{ACKNOWLEDGMENTS}

The authors would like to thank The Deanship of Scientific Research, Taif University.

\section{CONFLICT OF INTEREST}

The authors declare that there is no conflict of interest.

\section{AUTHORS' CONTRIBUTION}

$A G, M M F, A K I$ and $M H H$ involved in the design of the research. $A G$ and $M H H$ designed the experimental procedure. AG performed the molecular cloning and gene expression experiments. MMF and AKI involved in SDS-PAGE experiment. AG wrote the original draft. MMF, AKI, $\mathrm{MHH}, \mathrm{SA}$ and WFA involved in analysis the data. MMF, AKI, MHH, SA and WFA reviewed and edited the final version. All authors agreed with the final version of the manuscript.

\section{FUNDING}

The authors would like to thank The Deanship of Scientific Research, Taif University for funding this research [grant number1-438-5947].

\section{ETHICS STATEMENT}

This article does not contain any studies with human participants or animals performed by any of the authors.

\section{DATA AVAILABILITY}

All datasets analyzed in the study are involved in the manuscript and existing as tables and figures.

\section{REFERENCES}

1. Hosoya-Matsuda N, Motohashi K, Yoshimura $\mathrm{H}$, et al. Anti-oxidative stress system in cyanobacteria: Significance of type II peroxiredoxin and the role of 1-Cys peroxiredoxin in Synechocystis sp. strain PCC 6803. J Biol Chem. 2005;280(1):840-846. https://doi. org/10.1074/jbc.M411493200

2. Dietz KJ. Plant Peroxiredoxins. Annu Rev Plant Physiol Plant Mol Biol. 2003;54:93-107. https://doi. org/10.1146/annurev.arplant.54.031902.134934

3. Storz G, Jacobson FS, Tartaglia LA, Morgan RW, Silveira LA, Ames BN. An alkyl hydroperoxide reductase induced by oxidative stress in Salmonella typhimurium and Escherichia coli: genetic characterization and cloning of ahp. J Bacteriol. 1989;171(4):2049-2055. https://doi.org/10.1128/JB.171.4.2049-2055.1989

4. Jacobson, FS, Morgan RW, Christman MF, Ames BN. An alkyl hydroperoxide reductase from Salmonella typhimurium involved in the defense of DNA against oxidative damage. Purification and properties. $J$ Biol Chem. 1989;264:1488-1496. 
5. Perelman A, Uzan A, Hacohen D, Schwarz R. Oxidative stress in Synechococcus sp. strain PCC 7942: various mechanisms for $\mathrm{H}_{2} \mathrm{O}_{2}$ detoxification with different physiological roles. J Bacteriol. 2003;185:3654-3660. https://doi.org/10.1128/JB.185.12.3654-3660.2003

6. Kaneko $\mathrm{T}$, Sato $\mathrm{S}$, Kotani $\mathrm{H}$, et al. Sequence analysis of the genome of the unicellular cyanobacterium Synechocystis sp. strain PCC6803. II Sequence determination of the entire genome and assignment of potential protein-coding regions. DNA Res. 1996;3:109136. https://doi.org/10.1093/dnares/3.3.109

7. Kobayashi M, Ishizuka T, Katayama M, et al. Response to oxidative stress involves a novel peroxiredoxin gene in the unicellular cyanobacterium Synechocystis sp. PCC 6803. Plant Cell Physiol. 2004;45:290-299. https:// doi.org/10.1093/pcp/pch034

8. Latifi A, Ruiz M, Jeanjean R, Zhang CC. PrxQ-A, a member of the peroxiredoxin $Q$ family, plays a major role in defense against oxidative stress in the cyanobacterium Anabaena sp. strain PCC7120. Free Radic Biol Med. 2007;42:424-431. https://doi. org/10.1016/j.freeradbiomed.2006.11.011

9. Perez-Perez ME, Mata-Cabana A, Sanchez-Riego AM, Lindahl M, Florencio FJ. A Comprehensive Analysis of the Peroxiredoxin Reduction System in the Cyanobacterium Synechocystis sp. Strain PCC 6803 Reveals that All Five Peroxiredoxins Are Thioredoxin Dependent. J Bacteriol. 2009;191(24):7477-7489. https://doi.org/10.1128/JB.00831-09

10. Singh AK, Elvitigala $T$, Bhattacharyya-Pakrasi $M$, Aurora R, Ghosh B, Pakrasi HB. Integration of carbon and nitrogen metabolism with energy production is crucial to light acclimation in the cyanobacterium Synechocystis. Plant Physiol. 2008;148:467-478. https://doi.org/10.1104/pp.108.123489

11. Gaber A, Yoshimura K, Tamoi M, Takeda T, Nakano $Y$, Shigeoka S. Induction and Functional Analysis of Two Reduced Nicotinamide Adenine Dinucleotide Phosphate-Dependent Glutathione PeroxidaseLike Proteins in Synechocystis PCC 6803 during the Progression of Oxidative Stress. Plant Physiol. 2004;136(1):2855-2861. https://doi.org/10.1104/ pp.104.044842

12. Gaber A, Yoshimura K, Yamamoto T, et al. Glutathione peroxidase-like protein of Synechocystis PCC 6803 confers tolerance to oxidative and environmental stresses in transgenic Arabidopsis. Physiol Plant. 2006;128:251-262. https://doi.org/10.1111/j.13993054.2006.00730.x

13. Gao H, Xu X. The Cyanobacterial NAD Kinase Gene sll1415 Is Required for Photoheterotrophic Growth and Cellular Redox Homeostasis in Synechocystis sp. Strain PCC 6803. J Bacteriol. 2012;194(12):218-224. https://doi.org/10.1128/JB.05873-11

14. Allen MM. Simple conditions for the growth of unicellular blue-green algae on plates. J Phycol. 1968;4:1-4. https://doi.org/10.1111/j.1529-8817.1968.tb04667.x

15. Gaber A, Hassan MM, El- Awady MA. The overproduction of Synechocystis sp. PCC 6803 heatshock protein (SII0170) protects Escherichia coli against high-temperature stress. Biotechnol Equip. 2015;29(6):1201-1207. https://doi.org/10.1080/131

\subsubsection{6}

16. Thompson JD, Higgins DG, Gibson TJ. CLUSTALW: improving the sensitivity of progressive multiple sequence alignment through sequence weighting, position specific gap penalties and weight matrix choice. Nucleic Acids Res. 1994;22:4673-4680. https:// doi.org/10.1093/nar/22.22.4673

17. Lushchak, VI. Free radicals, reactive oxygen species, oxidative stress and its classification. Chem Biol Interact. 2014;224:164-175. https://doi.org/10.1016/j. cbi.2014.10.016

18. Brehelin C, Meyer EH, de Souris JP, Bonnard G, Meyer $Y$. Resemblance and dissemblance of Arabidopsis type II peroxiredoxins: similar sequences for divergent gene expression, protein localization, and activity. Plant Physiol. 2003;132(4):2045-2057. https://doi. org/10.1104/pp.103.022533

19. Sharapov MG, Ravin VK, Novoselov VI. Peroxiredoxins as Multifunctional Enzymes. Mol Biol. 2014;48(4):520545. https://doi.org/10.1134/S0026893314040128

20. Rhee SG, Woo HA, Kil IS, Bae SH. Peroxiredoxin Functions as a Peroxidase and a Regulator and Sensor of Local Peroxides. J Biol Chem. 2012;287(7):44034410. https://doi.org/10.1074/jbc.R111.283432

21. Kim K, Kim IH, Lee KY, Rhee SG, Stadtman, ER. The isolation and purification of a specific "protector" protein which inhibits enzyme inactivation by a thiol/ $\mathrm{Fe}(\mathrm{III}) / \mathrm{O} 2$ mixed-function oxidation system. J Biol Chem. 1988;263:4704-4711.

22. Chae HZ, Kim IH, Kim K, Rhee SG. Cloning, sequencing, and mutation of thiol-specific antioxidant gene of $S$. cerevisiae. J Biol Chem. 1993;268:16815-16821.

23. Chae HZ, Robison K, Poole LB, Church G, Storz $G$, Rhee SG. Cloning and sequencing of thiolspecific antioxidant from mammalian brain: alkyl hydroperoxide reductase and thiol-specific antioxidant define a large family of antioxidant enzymes. Proc Natl Acad Sci USA. 1994;91:7017-7021. https://doi. org/10.1073/pnas.91.15.7017

24. Rhee SG, Woo HA. Multiple functions of peroxiredoxins: peroxidases, sensors and regulators of the intracellular messenger $\mathrm{H}_{2} \mathrm{O}_{2}$, and protein chaperones. Antioxid Redox Signal. 2011;15:781-794. https://doi. org/10.1089/ars.2010.3393

25. Kim HS, Kang SW, Rhee SG, Clerch LB. Rat lung peroxiredoxins I and II are differentially regulated during development and by hyperoxia. Am J Physiol Lung Cell Physiol. 2001;280:1212-1217. https://doi. org/10.1152/ajplung.2001.280.6.L1212

26. Ito T, Kimura S, Seto K, et al. Peroxiredoxin I plays a protective role against UVA irradiation through reduction of oxidative stress. J Dermatol Sci. 2013;12:S0923-S1811.

27. Lee K, Park JS, Kim YJ, et al. Differential expression of Prx I and II in mouse testis and their up-regulation by radiation. Biochem Biophys Res Commun. 2002;296:337-342. https://doi.org/10.1016/S0006291X(02)00801-X

28. Kumar S, Stecher G, Tamura K. MEGA7: Molecular Evolutionary Genetics Analysis version 7.0 for bigger datasets. Mol Biol Evol. 2016; 33:1870-1874. https:// doi.org/10.1093/molbev/msw054 